\title{
Evaluating the Solar Slowly Varying Component at C-Band Using Dual- and Single-Polarization Weather Radars in Europe
}

\author{
M. Gabella, ${ }^{1}$ A. Huuskonen, ${ }^{2}$ M. Sartori, ${ }^{1}$ I. Holleman, ${ }^{3}$ M. Boscacci, ${ }^{1}$ and U. Germann ${ }^{1}$ \\ ${ }^{1}$ MeteoSwiss, Locarno-Monti, Switzerland \\ ${ }^{2}$ Finnish Meteorological Institute, Helsinki, Finland \\ ${ }^{3}$ Faculty of Science, Radboud University, Nijmegen, Netherlands
}

Correspondence should be addressed to M. Gabella; marco.gabella@meteoswiss.ch

Received 30 March 2017; Revised 11 August 2017; Accepted 23 August 2017; Published 8 October 2017

Academic Editor: Hisayuki Kubota

Copyright (C) 2017 M. Gabella et al. This is an open access article distributed under the Creative Commons Attribution License, which permits unrestricted use, distribution, and reproduction in any medium, provided the original work is properly cited.

\begin{abstract}
Six C-band weather radars located in Europe (Finland, Netherlands, and Switzerland) have been used to monitor the slowly varying solar emission, which is an oscillation with an amplitude of several decibels and a period of approximately 27 days. It is caused by the fact that the number of active regions that enhance the solar radio emission with respect to the quiet component, as seen from Earth, varies because of the Sun's rotation about its axis. The analysis is based on solar signals contained in the polar volume data produced during the operational weather scan strategy. This paper presents hundreds of daily comparisons between radar estimates and the Sun's reference signal, during the current active Sun period (year 2014). The Sun's reference values are accurately measured by the Dominion Radio Astrophysical Observatory (DRAO) at S-band and converted to C-band using a standard DRAO formula. Vertical and horizontal polarization receivers are able to capture the monthly oscillation of the solar microwave signal: the standard deviation of the log-transformed ratio between radars and the DRAO reference ranges from 0.26 to $0.4 \mathrm{~dB}$. A larger coefficient (and a different value for the quiet Sun component) in the standard formula improves the agreement.
\end{abstract}

\section{Introduction}

Meteorological radars are designed for detecting, monitoring, and tracking complex and highly variable precipitation fields in four dimensions. In Europe, most of these radars are operating at C-band ( $\sim 5 \mathrm{~cm}$ wavelength). In this paper, we show that modern meteorological radars are able to detect and monitor both the quiet and the slowly variable component of the solar signal.

The solar atmosphere continuously emits radio emission at all wavelengths. At every wavelength, there is a welldefined minimum, which is reached when the Sun is free of spots; this is called the quiet emission of the Sun. The presence of Sunspots enhances the radio emission, producing a slowly variable component. As solar magnetic activity ebbs and flows over its ten/thirteen-year cycle, varying numbers of active regions appear on the solar disc: Sunspots are the most obvious visible features in active regions. Because of the Sun's rotation about its axis (variable, as a function of latitude), the number of active regions that enhances the radio emission with respect to the quiet component, as seen from Earth, varies. The result is an oscillation, with an amplitude of several decibels at both $5 \mathrm{~cm}$ and $10.7 \mathrm{~cm}$ wavelengths and a period of approximately 27 days. The main objective of this paper is the comparison between the amplitudes of such oscillation observed at $5 \mathrm{~cm}$ (within the so-called Cband of the electromagnetic spectrum) and the reference one, which is measured at $10.7 \mathrm{~cm}$ (S-band). This paper does not intend to introduce weather radar estimates of the solar flux as an additional product for the solar community; rather, it just aims at describing the possibility of their use. A detailed description of S-band measurements and C-band daily retrieved values is presented in Section 3, just after a short description given in Section 2 regarding the Sun properties in terms of radio emission. Section 4 presents the dataset, which has been acquired during a recent active Sun period (for most radars, the same 100 days in the year 2014) and the scores used for assessing the agreement in 
quantitative terms. Section 5 presents the agreement between radar-received horizontal and vertical polarization and the reference unpolarized values. A short summary, conclusions, and outlook are presented in Section 6.

\section{Solar Radio Emission Properties}

The Sun's radio output varies considerably in time and frequency. Solar conditions are often described by the terms "quiet," "disturbed," and "active." The quiet solar conditions of the Sun occur in the absence of localized sources and are due to the presence of thermal emissions in the solar atmosphere. The values of the minima observed since 1949 provide information about the mean quiet Sun during the last period of approximately 55 years. However, since there is always some magnetic activity, it is not clear what constitutes a truly "quiet Sun." The disturbed solar conditions of the Sun have an extra component, which originates from bright regions. Also called the slowly varying component, it exhibits a nominal 27-day periodicity, which is synchronous with the solar rotations. As stated in the Introduction, the spectral range over which the slowly varying solar component is significant, compared to the quiet radio flux, is between 57 and $2 \mathrm{~cm}$; such a component is most effectively detected around $10 \mathrm{~cm}$. This property of the emissions, together with the entirely fortuitous choice of $10.7 \mathrm{~cm}$ for long-term solar monitoring programs, starting from the beginning of the last century, has been a major factor in making the $10.7 \mathrm{~cm}$ solar flux a milestone index of solar activity. An example of the close correlation between the annually averaged radio emission at $10.7 \mathrm{~cm}$ and the Sunspot number can be found in Tapping and Zwaan [1]. Another remarkable correlation is that pertaining to the total solar irradiation (the largest part of which is by far within the UV, visible, and near-infrared spectral region) (see, e.g., [2]).

The quiet solar radio flux has three distinct spectral regions for microwave frequencies:

(i) At frequencies lower than $350 \mathrm{MHz}$, the spectrum approximately follows the $f^{2}$ Rayleigh-Jeans law and corresponds to the one-million-Kelvin-degree quiet corona.

(ii) At frequencies higher than $6 \mathrm{GHz}$, the emission mainly originates in the chromosphere, following the $f^{2}$ Rayleigh-Jeans approximation of a blackbody of 6000 to $10000 \mathrm{~K}$.

(iii) In between, there is a transition from predominantly chromospheric to transition region radiation and finally to predominantly coronal emission.

Quiet and slowly varying emissions are both thermal radiation: the brightness temperature, which is proportional to the emittance in the Rayleigh-Jeans approximation of the Planck law, corresponds to an average temperature weighted over a given wavelength range. The thermal emission altitude in the solar atmosphere depends on this law: the longer the wavelength, the higher the dominant altitude of the emission. Unlike nonthermal emissions, the brightness temperature of thermal emissions cannot exceed the plasma temperature.
The third or sporadic component is associated with an active Sun. Radio bursts, which are often associated with solar flares, may exceed quiet Sun levels by several dB. In these cases, thermal radiation is outshone, for a time of some milliseconds to hours, by much brighter emissions by a population of high-energy electrons that have a nonthermal energy distribution (flares). During intense Sunspot activity, the solar flux density and the apparent center of emission vary unpredictably. No flares have been observed by DRAO or the radars in Europe during the temporal period analyzed in this paper (from April 8 to September 15, 2014). More detailed information regarding the solar radio emission can be found, for instance, in Tapping [3] and Benz [4] and the cited literature therein.

\section{Dedicated Measurements of Solar Flux Density at S-Band and Meteorological Radar Observations at C-Band}

\subsection{Daily Measurements of Solar Flux at S-Band: The DRAO} Reference Signal. The solar flux density at a $10.7 \mathrm{~cm}$ wavelength is continuously monitored at the Dominion Radio Astrophysical Observatory (DRAO). This observatory is located near Penticton, British Columbia, Canada, and is characterized by low interference levels at the decimeter and centimeter wavelengths; the quality of the environment is maintained through strong local, provincial, and federal protection [3]. Daily observations were started in 1946 and have continued to the present day. The current solar flux at $10.7 \mathrm{~cm}, F_{10.7}$, can be obtained from the DRAO observatory website. Such values are corrected for atmospheric attenuation. In particular, they refer to precise measurements acquired three times a day. In summer, this takes place at 17, 20 (local noon), and 23 UTC. The hilly horizon and $50^{\circ} \mathrm{N}$ latitude cause the Sun to be too low for the first and last timings during the "winter season"; as a consequence, the flux determinations are made at 18, 20, and 22 UTC during "darker months." DRAO measurements last one hour (instead of a few seconds characterizing radar observations with a moving antenna during operational scan program); the long-integration period significantly reduces the noise.

In Section 4.1 of Gabella et al. [5], it has been shown that the three series of precise $10.7 \mathrm{~cm}$ measurements acquired by DRAO are in practice undistinguishable in terms of comparison with radar observations. In this paper, similar to Gabella et al. [5], the daily radar values presented in Section 3.3 will be evaluated against the 20 UTC dataset, which is used as the reference for quantitatively assessing the degree of agreement.

3.2. Converting DRAO Reference Values from S-Band to C$B$ and. Since the focus of this work is on relative and not on absolute calibration, it is feasible to use the $10.7 \mathrm{~cm}$ solar flux measured by the DRAO to estimate an expected flux density at the C-band: the $10.7 \mathrm{~cm}$ solar flux measurements are converted into other wavelengths with an uncertainty that depends on the difference in wavelength. In fact, a transformation equation that makes use of the remarkable 
TABLE 1: Estimate of the quiet solar flux density $q_{\lambda}$ at various wavelengths.

\begin{tabular}{lcc}
\hline Wavelength & $\begin{array}{c}\text { Value of } q_{\lambda} \text { according to } \\
\text { Tapping [3] }\end{array}$ & $\begin{array}{c}q_{\lambda} \text { according to Benz } \\
{[4]}\end{array}$ \\
\hline $30 \mathrm{~cm}$ & $45 \mathrm{sfu}$ & $41 \mathrm{sfu}$ \\
$20 \mathrm{~cm}$ & $49 \mathrm{sfu}$ & $48 \mathrm{sfu}$ \\
$10 \mathrm{~cm}$ & $68 \mathrm{sfu}$ & $69 \mathrm{sfu}$ \\
$8 \mathrm{~cm}$ & $76 \mathrm{sfu}$ & $82 \mathrm{sfu}$ \\
$6 \mathrm{~cm}$ & $102 \mathrm{sfu}$ & $107 \mathrm{sfu}$ \\
$3 \mathrm{~cm}$ & $255 \mathrm{sfu}$ & $275 \mathrm{sfu}$ \\
$2 \mathrm{~cm}$ & $495 \mathrm{sfu}$ & $574 \mathrm{sfu}$ \\
$1 \mathrm{~cm}$ & $1980 \mathrm{sfu}$ & $1862 \mathrm{sfu}$ \\
\hline
\end{tabular}

stability of the blackbody spectrum of the slowly varying component of solar activity can be used for this purpose. The equation is a simple linear transformation:

$$
F_{\lambda}=p_{\lambda} \cdot\left(F_{10.7}-q_{10.7}\right)+q_{\lambda},
$$

where $p_{\lambda}$ and $q_{\lambda}$ represent the scaling factor and the quiet Sun flux density for the desired wavelength, while $q_{10.7}$ is the quiet solar flux density at the $10.7 \mathrm{~cm}$ reference wavelength. The rationale behind such equation is described in Section 5 of Tapping [3]. From his Table 1 in Section 5, the scaling factor $p_{\lambda}$ at 5 and $6 \mathrm{~cm}$ is 0.71 and 0.73 , respectively; with simple linear interpolation, the scaling factor for the Swiss meteorological radar $(\lambda=5.5 \mathrm{~cm})$ can be estimated as $p=$ 0.715 .

From a physics viewpoint, the flux densities observed in radio astronomy are spectral irradiances; consequently, they are given in power per unit bandwidth and per unit equivalent collecting area of the antenna. Using mks units, these powers are very small: in radio astronomy, one flux unit corresponds to $10^{-26} \mathrm{~W} \mathrm{~m}^{-2} \mathrm{~Hz}^{-1}$. The Sun is a strong radio source and its flux density is often given in solar flux units (sfu), where $1 \mathrm{sfu}=10^{-22} \mathrm{~W} \mathrm{~m}^{-2} \mathrm{~Hz}^{-1}$. According to Tapping [3], the quiet solar flux density at $2.8 \mathrm{GHz}$ is $q_{10.7}=$ $64 \mathrm{sfu}$. It can be interesting to compare these estimates with those by another source, for instance, Benz [4]: figures for 8 frequencies (between $1 \mathrm{GHz}$ and $30 \mathrm{GHz}$ ) are listed in Table 1 . However, as pointed out by Tapping [6], there is no reliable way that measurements at $10.7 \mathrm{~cm}$ wavelength can be applied to calibration of antennas operating at other frequencies. Hence, multiwavelength measurements are needed to add context and weight to flux measurements at S-band.

\subsection{Horizontal and Vertical Polarization Radar Observations} at C-Band. The idea of using the solar signal detected by ground-based radars during the operational weather scan program has been presented in a series of papers: Huuskonen and Holleman [7] presented the use of solar signals to determine antenna pointing. Holleman et al. [8] extended the method towards quantitative electromagnetic power measurements, in order to monitor radar receiving chain stability. In the case of dual-polarization radars, which are able to measure both horizontal $(\mathrm{H})$ and vertical $(\mathrm{V})$ polarization, the same technique can be used to monitor the presence (and stability) of a residual differential reflectivity offset: the stability of differential radar reflectivity was investigated by Holleman et al. [9] during a period of quiet Sun emissions (three months in 2008) and by Gabella et al. [5] during high solar activity (the first seven months of 2014).

A complete description of the automated detection of Sun signatures in polar volume data of weather radar can be found in Huuskonen and Holleman [7]; a similar approach is used at MeteoSwiss: details are provided in Section 2 of Gabella et al. [5]. The number of daily Sun hits depends on the operational scan strategy, the season, and the latitude of the radar; orography may also influence the lowest elevations, which are anyhow discharged for the Swiss radars in order to minimize the influence of refractivity and attenuation. Note that atmospheric attenuation is corrected in the Finnish and Dutch radars. Unfortunately, Sun hits are practically never detected with the radar beam axis hitting the center of the solar disk: hence, the challenge is to retrieve, by means of the least square method and Gaussian fit applied to the main lobe of the antenna radiation pattern, the peak solar power that the radar would have received if the beam had hit the Sun's center $[7,10]$. This is a crucial aspect of the retrieval: to derive, out of several tens of measurements per day, the most representative daily value; note that the signal-to-noise ratio of each measurement varies as a function of the angular distance between the Sun and the antenna radiation pattern beam axis. As stated, the main lobe can be approximated with a Gaussian, which means a parabolic decrease of the signal-to-noise ratio expressed in $\mathrm{dB}$ as a function of the angular offset. For the high-sensitivity C-band systems (low receiver losses, equipped with low noise amplifiers, antenna half-power beamwidth better than $1^{\circ}$ ) used in this study, the log-transformed ratio between the Sun + noise and the noise value ranges between 9 and $10 \mathrm{~dB}$ only when the beam axis of the main lobe hits the center of the solar disk. However, on the one hand, all measurements were de facto performed with a poorer signal-to-noise ratio; on the other hand, the noise contribution varied significantly from hit to hit and from day to day. Part of this noise is subtracted, and another part is filtered by averaging for one daily value. Anyhow, as expected, the noise affecting radar estimates is much larger than the one affecting DRAO measurements, as shown in Figures 1 and 2.

\section{Description of the Instruments, Scores, and Selected Observation Periods}

4.1. Swiss Radars (Wavelength $=5.5 \mathrm{~cm}$ ). MeteoSwiss has recently renewed its weather radar network: antennamounted, fully digital receivers have been introduced into the current network [11]. Each system is equipped with two orthogonal receiving channels, which are able to measure vertical and horizontal linear polarization at the operating frequency in the $5433-5468 \mathrm{MHz}$ range. The scan strategy is a crucial element in the design of a radar network to be operated in a mountainous region. MeteoSwiss scan program consists of 20 elevation sweeps repeated every five minutes with angles of elevation between $-0.2^{\circ}$ and $40^{\circ}$; the 


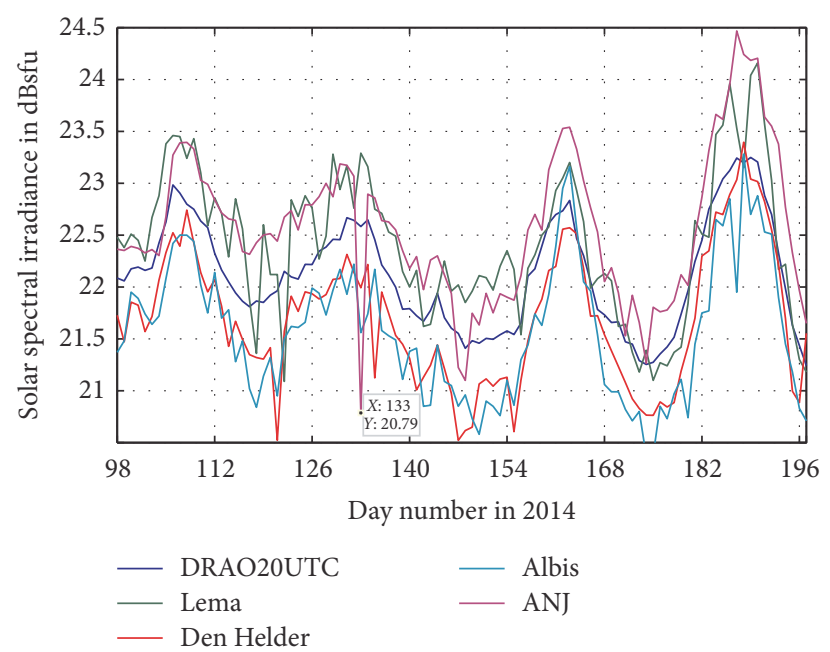

FIGURE 1: Daily variability of the horizontal polarization solar radio emission retrieved from four C-band weather radars in Europe from April 8 to July 16, 2014. The values from DRAO (20 UTC) are plotted for reference purposes: DRAO measurements are acquired at S-band using a 1-hour integration period and converted to C-band using (1).

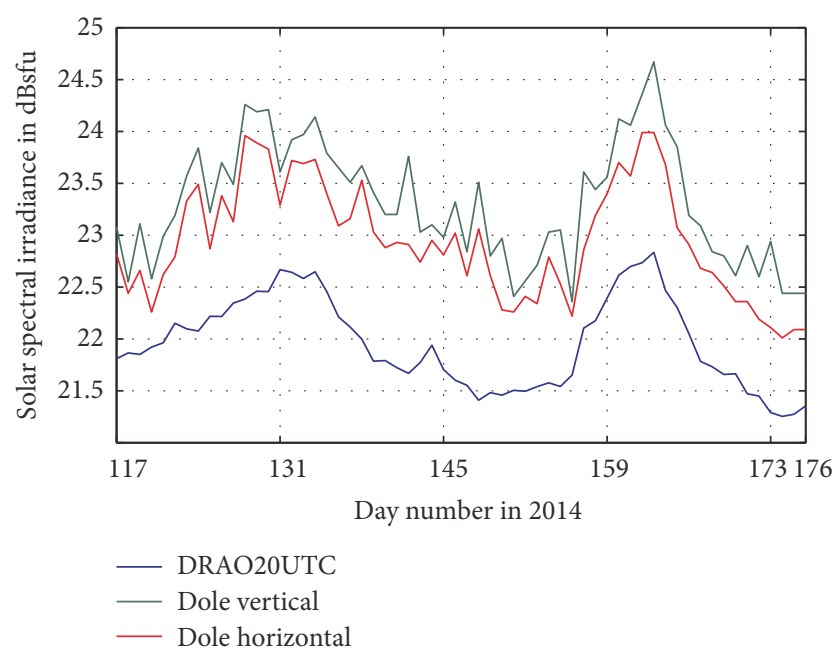

FIGURE 2: Daily variability of the vertical and horizontal polarization solar radio emission retrieved from the C-band weather radar located at La Dole in Switzerland. The 60-day observation period runs from April 27 to June 25.

products are updated every 2.5 minutes, taking advantage of an interleaved sweep pattern (for details, see Figure at page 45 in [11]). Three radar systems (out of the five installed) were available during the whole 2014: Lema (1625 m altitude, near Lugano; lat. $46.04^{\circ}$; lon. $8.83^{\circ}$ ), Albis (935 m, near Zurich; lat. $46.29^{\circ}$; lon. $\left.8.51^{\circ}\right)$, and Dole (1,681 m, near Geneva; lat. $46.43^{\circ}$; lon. $\left.6.10^{\circ}\right)$.

4.2. Dutch and Finnish Radars (Wavelength $=5.3 \mathrm{~cm}$ ). The Finnish Meteorological Institute operates a network of ten Cband Doppler weather radars, of which nine are polarimetric. Every 15 minutes, the radars perform 11 elevation sweeps with angles of elevation between $0.3^{\circ}$ and $45^{\circ}$. In the present study, data from the polarimetric Anjalankoski (ANJ) radar are presented. The ANJ site coordinates are lat. $60.90^{\circ}$, lon. $27.11^{\circ}$, and altitude $169 \mathrm{~m}$.

In 2014, the Royal Netherlands Meteorological Institute (KNMI) was operating a network of two identical C-band Doppler weather radars (horizontal polarization). Every 5 minutes, the radars perform 14 elevation sweeps with angles of elevation between $0.3^{\circ}$ and $25^{\circ}$. In the present study, horizontal polarization observations from these two sites are presented. The two radar sites are Den Helder (lat. $52.95^{\circ}$; lon. $4.79^{\circ}$; altitude $4 \mathrm{~m}$ ) and De Bilt (lat. $52.10^{\circ}$; lon. $5.18^{\circ}$; altitude $2 \mathrm{~m})$.

4.3. Scores for the Assessment of the Agreement. As stated in the Introduction, the present analysis is focused on the relative agreement and not on the absolute calibration of the radar receivers. In other words, emphasis is on the dispersion of the error around the mean. The error is defined as the log-transformed ratio (expressed in $\mathrm{dB}$ ) between the value measured by the radar receiver and that of the reference after conversion from S-band to C-band. This choice is due to the prevailing multiplicative nature of the errors that affect receiver measurements of the flux densities. The most intuitive score for assessing the relative agreement is the standard deviation of the error. Not surprisingly, when summarizing radar receiver performance during the quiet Sun period in 2008, Holleman et al. [8] have used the standard deviation of the log-transformed radar-to-DRAO daily ratios: for the Dutch De Bilt and Den Helder radars (46 days), the standard deviation was 0.14 and $0.17 \mathrm{~dB}$; for the Finnish Vimpeli and Luosto radars (44 days), it was 0.16 and $0.20 \mathrm{~dB}$. Another advantage of the standard deviation is that it is independent of any multiplicative bias that could affect the measurements. However, it is highly sensitive to any single large error (think, e.g., of a flare between 20 and 21 UTC, which would be detected by DRAO but certainly not by radars in Europe). Hence, we have opted for a second, robust, and informative score named "scatter" [12]. Its definition is strictly connected to the selected error distribution from a user and producer perspective. The cumulative error distribution is expressed as the cumulative weighted contribution to the normalized total amount ( $y$-axis) as a function of the log-transformed estimate/reference ratio ( $x$-axis). The scatter (also expressed in $\mathrm{dB}$ ) is defined as half the distance between the $84 \%$ and $16 \%$ percentiles of the weighted error distribution. The scatter as defined above is a robust measure of the dispersion of the error. It is independent of the bias and insensitive to outliers.

4.4. The Selected Sun Active Days in 2014 and Visual Comparison. Ideally, one would like to compare the agreement between the 6 European radars and the DRAO reference exactly during the same Sun active period. Furthermore, one would like to have a period that includes at least 3 or 4 Sun rotations (slowly varying component oscillations). However, as it will be seen in Section 5, we are facing agreement that is of the order of few tenths of $\mathrm{dB}$; this means that even a small $0.1 \mathrm{~dB}$ jump ("discontinuity") or drift in either the vertical or the horizontal channel of any radar would influence 
TABLE 2: Agreement between six radars in Europe and the DRAO reference in 2014.

\begin{tabular}{|c|c|c|c|c|}
\hline Radar & Polarization & Analyzed days & Scatter in $\mathrm{dB}$ & Stand. dev. in $\mathrm{dB}$ \\
\hline Den Helder (NL) & Horizontal & From 98 to 197 & $0.22 \mathrm{~dB}$ & $0.26 \mathrm{~dB}$ \\
\hline Albis $(\mathrm{CH})$ & Horizontal & From 98 to 197 & $0.23 \mathrm{~dB}$ & $0.26 \mathrm{~dB}$ \\
\hline ANJ (Suomi) & Horizontal & From 98 to 197 & $0.25 \mathrm{~dB}$ & $0.36 \mathrm{~dB}$ \\
\hline Dole $(\mathrm{CH})$ & Horizontal & From 117 to 176 & $0.26 \mathrm{~dB}$ & $0.27 \mathrm{~dB}$ \\
\hline De Bilt (NL) & Horizontal & From 169 to 258 & $0.26 \mathrm{~dB}$ & $0.25 \mathrm{~dB}$ \\
\hline Lema $(\mathrm{CH})$ & Horizontal & From 98 to 197 & $0.36 \mathrm{~dB}$ & $0.36 \mathrm{~dB}$ \\
\hline Albis $(\mathrm{CH})$ & Vertical & From 98 to 197 & $0.25 \mathrm{~dB}$ & $0.27 \mathrm{~dB}$ \\
\hline ANJ (Suomi) & Vertical & From 98 to 197 & $0.24 \mathrm{~dB}$ & $0.40 \mathrm{~dB}$ \\
\hline Dole $(\mathrm{CH})$ & Vertical & From 117 to 176 & $0.29 \mathrm{~dB}$ & $0.31 \mathrm{~dB}$ \\
\hline Lema $(\mathrm{CH})$ & Vertical & From 98 to 197 & $0.37 \mathrm{~dB}$ & $0.40 \mathrm{~dB}$ \\
\hline
\end{tabular}

our investigation regarding the slowly varying component. Hence, we have to carefully select a period free of any kind of discontinuity or drift for both polarization channels (possible causes of small discontinuities or drifts go beyond the scope of this note).

For four radars, it was possible to select a 100-day-long common period having 4 maxima of the S-component: it lasted 100 days, from April 8 to July 16, 2014 (day of the year 98 and 197); the solar slowly varying component, as measured by DRAO and as seen by 4 horizontal channels, is shown in Figure 1. Note that solar flux units on the $y$-axis are logtransformed. In the present case, $s=10 \log \left(F / F_{0}\right)$ is used, where $F_{0}=1 \mathrm{sfu}=10^{-19} \mathrm{~mW} \cdot \mathrm{m}^{-2} \cdot \mathrm{Hz}^{-1}$ and $[s]=\mathrm{dBsfu}$. All 4 radars are able to capture and describe the "27-day" variability of the microwave signal emitted by the Sun; the DRAO reference curve, which refers to accurate 1-hour-long daily measurements with the antenna radiation pattern beam axis hitting the center of the disk, is obviously much smoother than radar daily estimates, which are retrieved by fitting tens of off-axis hits acquired during a fraction of a second. As pointed out by Gabella et al. [5] in Section 4.3, such random errors (sort of "daily jumps") affecting the radars curves can easily and effectively be removed by a running-median filter: even a "short-window," three-day running median is able to significantly reduce these daily radar fluctuations. For instance, the large ANJ jump on day 133 would be eliminated by the median filtering. Obviously a point like this has a strong influence on the standard deviation of the difference between the reference and ANJ: as it will be seen in Section 5.1 (Table 2), the standard deviation results to be $0.36 \mathrm{~dB}$; this is exactly why a score like the scatter was conceived (see Section 4.3): its value is, in fact, much smaller, which is $0.25 \mathrm{~dB}$, a value similar to the one for Den Helder and Albis. On the contrary, jumps and drops for the Lema (green curve) are much more numerous: hence, the scatter and standard deviation of Lema show similar figures (as it can be seen in Table 2, next section). If we analyze the correlation among the 5 curves shown in Figure 1 ( 98 degrees of freedom), we see that the best explained variance ( $87 \%$ ) is between Den Helder and DRAO, followed by ANJ and DRAO (84\%); the best correlation between two radars is slightly smaller: it involves Den Helder and ANJ, for which the explained variance is $81 \%$.

For Dole, it was possible to select a subperiod lasting only 60 days, namely, from April 27 (day 117) to June 25
TABLE 3: Agreement between the daily horizontal and vertical polarization solar signals as retrieved by four dual-polarization radars.

\begin{tabular}{lcc}
\hline Radar & Analyzed days in 2014 & Standard deviation in dB \\
\hline $\begin{array}{l}\text { Albis }(\mathrm{CH}) \\
\text { ANJ }\end{array}$ & From 98 to 197 & $0.06 \mathrm{~dB}$ \\
$\begin{array}{l}\text { Suomi) } \\
\text { Dole }(\mathrm{CH})\end{array}$ & From 98 to 197 & $0.08 \mathrm{~dB}$ \\
Lema $(\mathrm{CH})$ & From 117 to 176 & $0.08 \mathrm{~dB}$ \\
\hline
\end{tabular}

(day 176). This is because external noise has been intermittently affecting the Dole radar (until the end of 2016). Figure 2 shows that, during such period, two maxima of the slowly varying component have occurred: the blue curve represents again the DRAO reference, while we have opted in this case for visualizing both horizontal (red) and vertical (green) radar polarization observations. Although during these 60 days residual noise is smaller than usual, it still affects both radar channels (much more than the DRAO reference values); however, as stated, a three-day running median is able to significantly reduce daily radar fluctuations caused by noise. It is evident that the agreement between the two radar channels is better than that between $\mathrm{H}$ or $\mathrm{V}$ and DRAO: we will see in Tables 2 and 3 that standard deviation of the difference between Dole and DRAO is of the order of $0.3 \mathrm{~dB}$, while it is $0.08 \mathrm{~dB}$ between $\mathrm{H}$ and V. The interested reader can compare Figures 1 and 2 in this paper with Figure 1 of Gabella et al. [5], which shows the retrieved median daily value for the three Swiss radars from January 1 to August 12, 2015. For the De Bilt radar, the selected period is mostly not overlapping: it lasts 90 days and runs from June 18 to September 15, 2015.

\section{Quantitative Comparison between DRAO and Six Radars in Europe}

5.1. Daily Agreement between $H$ (or V) Polarization and the DRAO Reference. Results of the quantitative comparison between radars and the DRAO reference in terms of scatter and standard deviation (both in $\mathrm{dB}$ ) are listed in Table 2. Note that, for the Swiss radars, we have used $q_{5.5}=113$ and $p=0.715$ (as in [5]); for the Finnish and Dutch radars, we have followed Holleman et al. [8]: $q_{5.3}=126$ and $p=0.71$. 
TABLE 4: Agreement in terms of scatter in dB between six radars in Europe and the DRAO reference in 2014 as a function of value of the angular coefficient $p_{\lambda}$ in (1).

\begin{tabular}{|c|c|c|c|c|c|}
\hline Radar & $p_{\lambda}=0.715$ & $p_{\lambda}=0.80$ & $p_{\lambda}=0.88$ & $p_{\lambda}=0.95$ & $p_{\lambda}=1.00$ \\
\hline Den Helder (NL) & $0.22 \mathrm{~dB}$ & $0.19 \mathrm{~dB}$ & $0.16 \mathrm{~dB}$ & $0.15 \mathrm{~dB}$ & $0.16 \mathrm{~dB}$ \\
\hline Albis $(\mathrm{CH})$ & $0.23 \mathrm{~dB}$ & $0.22 \mathrm{~dB}$ & $0.21 \mathrm{~dB}$ & $0.22 \mathrm{~dB}$ & $0.24 \mathrm{~dB}$ \\
\hline ANJ (Suomi) & $0.25 \mathrm{~dB}$ & $0.24 \mathrm{~dB}$ & $0.24 \mathrm{~dB}$ & $0.23 \mathrm{~dB}$ & $0.23 \mathrm{~dB}$ \\
\hline Dole $(\mathrm{CH})$ & $0.26 \mathrm{~dB}$ & $0.25 \mathrm{~dB}$ & $0.26 \mathrm{~dB}$ & $0.28 \mathrm{~dB}$ & $0.28 \mathrm{~dB}$ \\
\hline De Bilt (NL) & $0.26 \mathrm{~dB}$ & $0.24 \mathrm{~dB}$ & $0.21 \mathrm{~dB}$ & $0.20 \mathrm{~dB}$ & $0.20 \mathrm{~dB}$ \\
\hline Lema $(\mathrm{CH})$ & $0.36 \mathrm{~dB}$ & $0.34 \mathrm{~dB}$ & $0.33 \mathrm{~dB}$ & $0.31 \mathrm{~dB}$ & $0.31 \mathrm{~dB}$ \\
\hline
\end{tabular}

The first six rows refer to horizontal polarization: 5 radars have similar good performances in terms of scatter; Lema is worse. As far as the Swiss radars are concerned, the comparison with Table 2 at page 55 of [5] shows that both scores respond to an "optimized" reduction (see Section 4.4) of the analyzed period from 224 to 100 days (Albis and Lema): on average, there is a reduction of $0.06(0.11) \mathrm{dB}$ for the scatter (standard deviation); the even larger ("optimized") reduction of the number of days for Dole results in a decrease of 0.19 (0.21) dB for the scatter (standard deviation).

As already stated, the large difference between the scatter and standard deviation of ANJ radar can easily be explained by the large $2 \mathrm{~dB}$ drop in the retrieved radar value that occurred on day 133 (see violet curve in Figure 1).

Regarding vertical polarization (last fourth lines), it is worth noting that performances are slightly worse than the horizontal one in terms of standard deviation and scatter; there is one exception, which is the scatter of ANJ.

5.2. The Role of Atmospheric Attenuation and Noise Subtraction. In this section, the focus is on the quantitative agreement between DRAO and each of the four radars with data during the same 100-day period. As stated in Section 3.3, a key aspect is the daily radar retrieval based on many solar hits, acquired at different angles of elevation and with different angular distances between the beam axis and the center of the Sun. The best approach is certainly the one described in Section 2, page 1705, of Huuskonen et al. [10], which is based on a five-parameter linear model to retrieve the power that the antenna would have received if the beam had hit the Sun's center. Performance scores listed in Table 2 are based on such an approach. If one is not interested in absolute calibration, but only in relative agreement, a much simpler approach can be the one used by Gabella et al. [5]: the median of, for instance, the strongest 21 hits per day is simply used in order to get a robust daily estimate. By doing so, as expected, the performance of the Swiss radars did not change significantly: the scatter of Albis (Lema) is $0.25(0.32) \mathrm{dB}$ instead of $0.23(0.36) \mathrm{dB}$. On the contrary, when using the median, the scatter of ANJ and Den Helder gets significantly worse: the latter deteriorates from $0.22 \mathrm{~dB}$ to $0.42 \mathrm{~dB}$, the former from $0.25 \mathrm{~dB}$ to $0.39 \mathrm{~dB}$. We think the main cause is the absence (in the Swiss data) of atmospheric correction, which in turn spoils the benefit of the fitting procedure. Another possible cause can be related to the unavoidable presence of unknown residual noise. The value of noise subtracted from the measured signal, in fact, may be quite different from the real noise affecting the radar observation of the Sun. In the Swiss radars, for instance, the subtracted noise value is the median of what is observed in every volume with an angle of elevation of $40^{\circ}$.

5.3. Relative Agreement between $H$ and $V$ Polarization (4 Dual-Polarization Radars). The issue of differential reflectivity calibration is crucial for successful applications of polarimetric weather radar (see, e.g., $[13,14]$ ). Thanks to Sun observations, it is possible to monitor the quality of the receiving chain using offline methods (e.g., [15]) or during the operational scan [9], which is the approach followed here. As seen in the previous section, both polarization types show similar performances compared to DRAO (horizontal is just slightly better); hence, it is not surprising that they look very similar, especially in terms of dispersion of the error around the mean. For this reason, for quantitatively assessing the agreement, the standard deviation is sufficient (the scatter is not needed): results are listed in Table 3. For all four radars, the agreement between daily retrieved horizontal and vertical observations is smaller than $0.1 \mathrm{~dB}$, even in terms of standard deviation. A comparison with Table 4 at page 57 of Gabella et al. [5] shows that such agreement does not depend on the length of the observation period: actually, with more than two hundred days, the standard deviation is even slightly smaller.

5.4. Increased Radars-DRAO Agreement Using a Larger Coefficient in (1). By looking at the $\sim 27$-day oscillation in Figures 1 and 2, it looks like the amplitude of the reference is even smaller than the retrieved curves using radar observations. This fact has suggested the idea of using a slightly larger coefficient in (1); four other values have been used and the agreement is evaluated in terms of scatter in $\mathrm{dB}$ : results are listed in Table 4 . The use of a larger coefficient causes a better agreement for all six radars. For Albis and ANJ (Dole), the improvement is very small; for Lema, De Bilt, and Den Helder, the scatter becomes $0.05,0.06$, and $0.07 \mathrm{~dB}$ smaller, respectively. As suggested by a reviewer, we have also tried to modify $q_{10.7}$ in addition to $p_{\lambda}$. For Den Helder, Albis, Dole, and De Bilt, a change of $q_{10.7}$ does not further improve the agreement. For ANJ (Lema), a value of 90 (74) sfu reduces the scatter from $0.23(0.31)$ to $0.20(0.30) \mathrm{dB}$.

\section{Summary, Conclusions, and Outlook}

In this paper, we discuss the use of the $5.5 \mathrm{~cm}$ solar flux density for routine monitoring of dual- and single-polarization 
weather radars during a common period of intense Sun activity in 2014: a daily comparison (during two/three months in 2014) between values retrieved from six radars in Europe and the DRAO reference shows standard deviation of the error between $0.26 \mathrm{~dB}$ and $0.40 \mathrm{~dB}$. This range of values is relatively close to what was obtained by Holleman et al. [8] during 44 days of a quiet solar period (2008). As far as the relative agreement between horizontal and vertical polarization is concerned, Holleman et al. [9] have found for the Trappes radar a standard deviation of the log-transformed $\mathrm{H}$-to-V daily ratios equal to $0.20 \mathrm{~dB}$ during a 3-month quiet Sun period in 2008. Modern polarimetric systems seem to outperform this benchmark: despite the solar activity occurring in 2014, the (100-day) standard deviation of Albis and Lema is $0.06 \mathrm{~dB}$, and that of ANJ and Dole (60 days) is $0.08 \mathrm{~dB}$. Note that, for the first 224 days in 2014, Gabella et al. [5] have obtained values ranging between $0.05 \mathrm{~dB}$ (Albis) and $0.06 \mathrm{~dB}$ (Lema and Dole). Recently, Huuskonen et al. [16] found a standard deviation as low as $0.02 \mathrm{~dB}$, which was found for the Finnish ANJ and LUO radars. These figures refer to a shorter observation period (April 2015) and an improved quality control to remove rain contaminated values in the solar hits. In Sections 4 and 5, it has been shown that radar-retrieved curves of solar flux are able to capture and describe the "27-day period" oscillation of the microwave signal emitted by the Sun, which is the so-called slowly varying component. We conclude that the analysis of solar signals acquired during operational scan program of weather radar is useful for monitoring receivers stability and checking the quality of differential radar reflectivity during not only quiet but also active solar periods.

In Switzerland, starting from the beginning of 2015, we have also implemented an analysis directly based on $I^{2}+$ $Q^{2}$ raw data, which is received power using a number of pulses not necessarily limited to $1^{\circ}$ in azimuth, in addition to the current one, which is based on radar reflectivity values stored directly in polar volume data $\left(1^{\circ}\right.$ half-power beamwidth). We are confident that future analysis based on this novel technique (by increasing also the number of radars and years) will allow us to better characterize the slowly varying component at $5.5 \mathrm{~cm}$. Noticeably, we have also implemented a complementary technique aiming at the absolute calibration of dual-polarization receivers: in order to maximize the signal-to-noise ratio, the radar receiver has to be offline for a few minutes during the tracking of the Sun in order to have the antenna beam axis pointing towards the center of the Sun [17]. Radar observations of the solar signal are performed on demand in fair weather conditions. In this way, wet-radome attenuation and attenuation along the path are avoided. Preliminary results were so encouraging that MeteoSwiss decided to implement a fast, semiautomated Sun-tracking technique, which has since then also been applied to the other four MeteoSwiss operational C-band radars [18]. The main disadvantage of such a Sun-tracking technique is that it requires the operational weather radar to be offline for a few minutes. Recently, the method has been successfully applied at X-band [19], despite the smaller signal-to-noise ratio and amplitude of the slowly varying component.

\section{Conflicts of Interest}

The authors declare no conflicts of interest.

\section{References}

[1] K. F. Tapping and C. Zwaan, "Sources of the slowly-varying component of solar microwave emission and their relationship with their host active regions," Solar Physics, vol. 199, no. 2, pp. 317-344, 2001.

[2] K. F. Tapping, D. Boteler, P. Charbonneau, A. Crouch, A. Manson, and H. Paquette, "Solar magnetic activity and total irradiance since the maunder minimum," Solar Physics, vol. 246, no. 2, pp. 309-326, 2007.

[3] K. F. Tapping, "Antenna Calibration Using the $10.7 \mathrm{~cm}$ Solar Flux," 2015, http://www.k5so.com/RadCal_Paper.pdf.

[4] A. O. Benz, "Radio emission of the quiet Sun," in LandoltBörnstein Numerical Data and Functional Relationships in Science and Technology, in Chief: W. Martienssen, GroupVI/4 Astronomy, J. E. Trümper, Ed., p. 14, Astronomy, Astrophysics and Cosmology, 2009.

[5] M. Gabella, M. Sartori, M. Boscacci, and U. Germann, "Vertical and horizontal polarization observations of slowly varying solar emissions from operational swiss weather radars," Atmosphere, vol. 6, no. 1, pp. 50-59, 2015.

[6] K. F. Tapping, "The $10.7 \mathrm{~cm}$ solar radio flux (F10.7)," Space Weather, vol. 11, no. 7, pp. 394-406, 2013.

[7] A. Huuskonen and I. Holleman, "Determining weather radar antenna pointing using signals detected from the sun at low antenna elevations," Journal of Atmospheric and Oceanic Technology, vol. 24, no. 3, pp. 476-483, 2007.

[8] I. Holleman, A. Huuskonen, M. Kurri, and H. Beekhuis, "Operational monitoring of weather radar receiving chain using the sun," Journal of Atmospheric and Oceanic Technology, vol. 27, no. 1, pp. 159-166, 2010.

[9] I. Holleman, A. Huuskonen, R. Gill, and P. Tabary, "Operational monitoring of radar differential reflectivity using the sun," Journal of Atmospheric and Oceanic Technology, vol. 27, no. 5, pp. 881-887, 2010.

[10] A. Huuskonen, M. Kurri, H. Hohti, H. Beekhuis, H. Leijnse, and I. Holleman, "Radar performance monitoring using the angular width of the solar image," Journal of Atmospheric and Oceanic Technology, vol. 31, no. 8, pp. 1704-1712, 2014.

[11] U. Germann, M. Boscacci, M. Gabella, and M. Sartori, "Radar design for prediction in the Swiss Alps," Meteorol. Tech. Int, 2015.

[12] U. Germann, G. Galli, M. Boscacci, M. Bolliger, and M. Gabella, "Quantitative precipitation estimation in the Alps: where do we stand?" in In Proceedings of the 3rd European Conf. on Radar in Meteorology and Hydrology (ERAD2004), Visby, Sweden, September 2004.

[13] A. V. Ryzhkov, S. E. Giangrande, V. M. Melnikov, and T. J. Schuur, "Calibration issues of dual-polarization radar measurements," Journal of Atmospheric and Oceanic Technology, vol. 22, no. 8, pp. 1138-1155, 2005.

[14] A. Illingworth, "Improved Precipitation Rates and Data Quality by Using Polarimetric Measurements," in Weather Radar, Physics of Earth and Space Environments, pp. 130-166, Springer Berlin Heidelberg, Berlin, Heidelberg, 2004.

[15] J. F. Pratte and D. G. Ferraro, "Automated solar gain calibration," in In Proceedings of the 24th Conference on Radar Meteorology, pp. 619-622, Tallahassee, FL, USA, 1989. 
[16] A. Huuskonen, M. Kurri, and I. Holleman, "Improved analysis of solar signals for differential reflectivity monitoring," Atmospheric Measurement Techniques, vol. 9, no. 7, pp. 3183-3192, 2016.

[17] M. Gabella, "Checking absolute calibration of vertical and horizontal polarization weather radar receivers using the solar flux," Journal of Electrical Engineering, vol. 3, pp. 163-169, 2015.

[18] M. Gabella, M. Boscacci, M. Sartori, and U. Germann, "Calibration accuracy of the dual-polarization receivers of the C-band swiss weather radar network," Atmosphere, vol. 7, no. 6, article no. 76, 2016.

[19] M. Gabella and A. Leuenberger, "Dual-Polarization Observations of Slowly Varying Solar Emissions from a Mobile X-Band Radar," Sensors, vol. 17, 15 pages, 2017. 

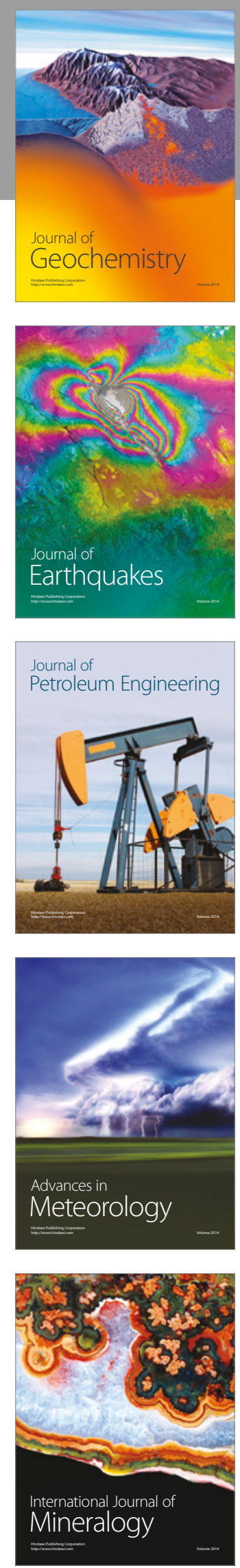
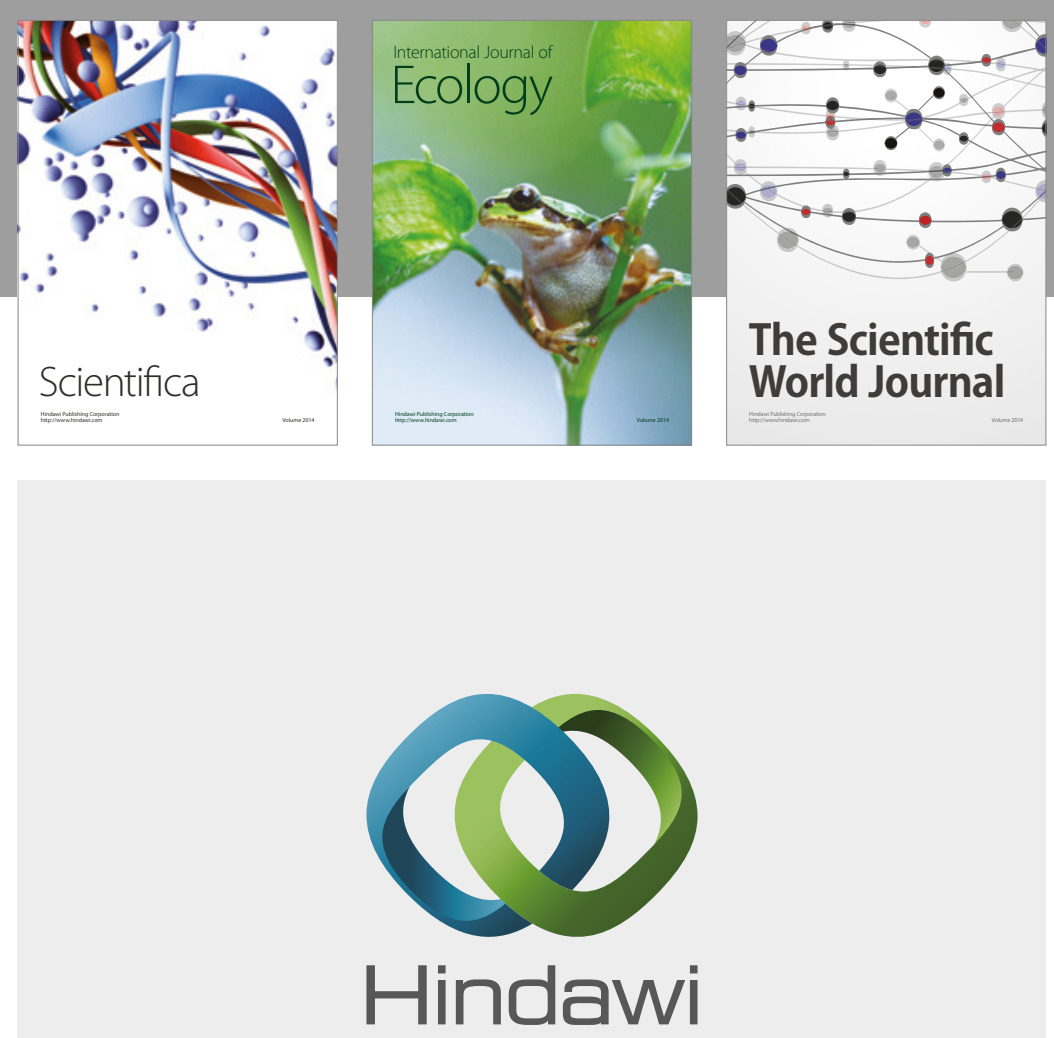

Submit your manuscripts at

https://www.hindawi.com
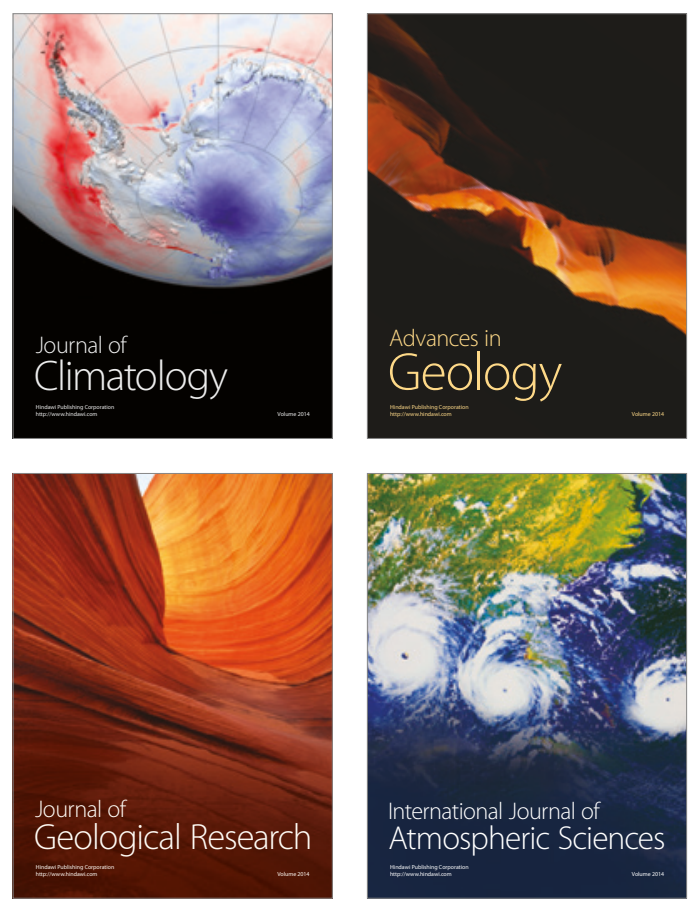

The Scientific

World Journal
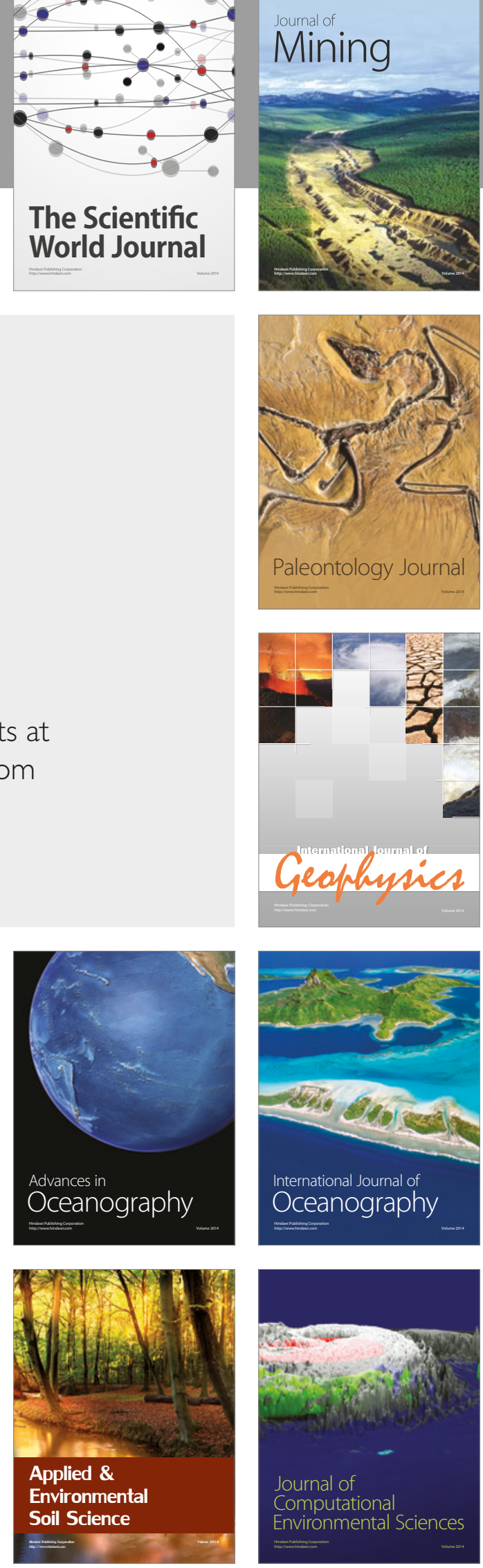